-8.0 to -26.0 ). Both components of the UAS7 (i.e., pruritus severity and wheal size) decreased to a similar extent. No drug-related adverse events were reported during the study. Similar efficacy results were observed in the initial study that assessed omalizumab (an anti-IgE antibody) for the treatment of chronic spontaneous urticaria. ${ }^{5}$ In addition, the percentage of patients who had a complete response in our study was similar to that in the omalizumab study. ${ }^{5}$

The prolonged clinical effect of benralizumab in the patients who had a complete response after discontinuation of the treatment may be explained by a modification of the biologic pathways that are important for promoting persistent cutaneous inflammation associated with chronic spontaneous urticaria. These observations support the use of benralizumab in the treatment of chronic spontaneous urticaria that is unresponsive to second-generation $\mathrm{H}_{1}$-antihistamines and provide evidence of a pathogenic role for infiltrating eosinophils. Therefore, a double-blind, placebo-controlled trial in a large patient population is warranted.
Jonathan A. Bernstein, M.D.

Umesh Singh, M.D., Ph.D.

Marepalli B. Rao, Ph.D.

University of Cincinnati College of Medicine

Cincinnati, $\mathrm{OH}$

bernstja@ucmail.uc.edu

and Others

A complete list of authors is available with the full text of this letter at NEJM.org.

Supported by AstraZeneca.

Disclosure forms provided by the authors are available with the full text of this letter at NEJM.org.

1. Zuberbier T, Aberer W, Asero R, et al. The EAACI/GA²LEN/ EDF/WAO guideline for the definition, classification, diagnosis and management of urticaria. Allergy 2018;73:1393-414.

2. Caproni M, Volpi W, Macchia D, et al. Infiltrating cells and related cytokines in lesional skin of patients with chronic idiopathic urticaria and positive autologous serum skin test. Exp Dermatol 2003;12:621-8.

3. Hawro T, Ohanyan T, Schoepke N, et al. The urticaria activity score - validity, reliability, and responsiveness. J Allergy Clin Immunol Pract 2018;6(4):1185-1190.e1.

4. Bracken SJ, Abraham S, MacLeod AS. Autoimmune theories of chronic spontaneous urticaria. Front Immunol 2019;10:627.

5. Kaplan AP, Joseph K, Maykut RJ, Geba GP, Zeldin RK. Treatment of chronic autoimmune urticaria with omalizumab. J Allergy Clin Immunol 2008;122:569-73.

DOI: 10.1056/NEJMc2016395

\title{
Amoxicillin Course Length or Use in Childhood Pneumonia in Underserved Areas
}

TO THE EDITOR: The trials by Ginsburg et al. ${ }^{1}$ and Jehan et al. ${ }^{2}$ (July 2 issue) question the recommended treatment of nonsevere pneumonia in children younger than 5 years of age. The trial conducted by Ginsburg et al. in Malawi showed that a 3-day course of amoxicillin was noninferior to a 5-day course, despite the former regimen having been shown to be insufficient in patients with radiologically confirmed pneumonia in a previous trial in Israel. ${ }^{3}$ The other trial, conducted by Jehan et al. in Pakistan (Randomized Trial of Amoxicillin Versus Placebo for [Fast Breathing] Pneumonia [RETAPP]), did not show noninferiority of placebo to a 3-day course of amoxicillin in patients with fast-breathing pneumonia.

More than $93 \%$ of the Pakistani children who had been randomly assigned to receive placebo recovered rapidly without relapse. The World Health Organization (WHO) criteria for diagnos- ing pneumonia probably capture a high proportion of viral illness, ${ }^{4}$ and both trials are limited by the lack of radiologic or laboratory assessment.

The identified predictors of treatment failure could guide new diagnostic definitions. A subgroup analysis of treatment-failure rates among nonvaccinated children would also be helpful, given that both trials reported vaccine coverage that was up to $30 \%$ lower than the average national rates. $^{5}$

The effect of overtreatment on the community should not be neglected in the era of increasing antimicrobial multiresistance. Therefore, the critical question is more likely to be "Is this bacterial pneumonia?" than "Should the treatment be shortened?"

\section{Laure F. Pittet, M.D., Ph.D.}

Murdoch Children's Research Institute

Parkville, VIC, Australia

laure.pittet@mcri.edu.au 
Seilesh Kadambari, M.D., Ph.D.

Yara-Natalie Abo, M.D.

Royal Children's Hospital Melbourne

Parkville, VIC, Australia

No potential conflict of interest relevant to this letter was reported.

1. Ginsburg A-S, Mvalo T, Nkwopara E, et al. Amoxicillin for 3 or 5 days for chest-indrawing pneumonia in Malawian children. N Engl J Med 2020;383:13-23.

2. Jehan F, Nisar I, Kerai S, et al. Randomized trial of amoxicillin for pneumonia in Pakistan. N Engl J Med 2020;383: 24-34.

3. Greenberg D, Givon-Lavi N, Sadaka Y, Ben-Shimol S, Bar-Ziv J, Dagan R. Short-course antibiotic treatment for communityacquired alveolar pneumonia in ambulatory children: a doubleblind, randomized, placebo-controlled trial. Pediatr Infect Dis J 2014;33:136-42.

4. Pneumonia Etiology Research for Child Health (PERCH) Study Group. Causes of severe pneumonia requiring hospital admission in children without HIV infection from Africa and Asia: the PERCH multi-country case-control study. Lancet 2019;394: 757-79.

5. Word Health Organization. Pneumococcal conjugate 3rd dose (PCV3) immunization coverage (https://www.who.int/gho/ immunization/pneumococcal/en/).

DOI: 10.1056/NEJMc2026544

DRS. GINSBURG AND MAY REPLY: We agree with Pittet et al. that the WHO guidelines for diagnosing pneumonia capture a high proportion of viral illness and that we were not able to assess this in our trial. We also agree that strategies to justify antibiotic treatment in children who are unlikely to have a bacterial infection are desirable.

We sought to develop a prognostic risk score to aid decision making about antibiotic treatment using a database of records from children with fast-breathing pneumonia (diagnosed according to WHO criteria) who received placebo in another randomized, controlled trial that we conducted in Malawi; however, the model had insufficient discrimination to be appropriate for clinical application..$^{1,2}$ Given that the majority of children in our trial population had inadequate and inconsistent vaccination documentation, we were unable to assess the effect of vaccination on pneumonia treatment failure. ${ }^{3}$

Adequate, rapid, point-of-care diagnostic tests are lacking for pneumonia and for differentiating viral from bacterial disease. Because treatment of pneumonia is critical for saving lives and the emergence of antimicrobial resistance is not merely a threat but a reality, the appropriate duration of antibiotic treatment remains a critical question of global concern.

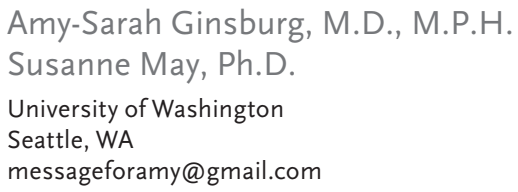

Since publication of their article, the authors report no further potential conflict of interest.

1. Ginsburg AS, Mvalo T, Nkwopara E, et al. Placebo vs amoxicillin for nonsevere fast-breathing pneumonia in Malawian children aged 2 to 59 months: a double-blind, randomized clinical noninferiority trial. JAMA Pediatr 2019;173:21-8.

2. McCollum ED, Brown SP, Nkwopara E, Mvalo T, May S, Ginsburg AS. Development of a prognostic risk score to aid antibiotic decision-making for children aged 2-59 months with World Health Organization fast breathing pneumonia in Malawi: an Innovative Treatments in Pneumonia (ITIP) secondary analysis. PLoS One 2019;14(6):e0214583.

3. Gadama D, Mvalo T, Ginsburg AS. Haemophilus influenzae type $\mathrm{b}$ and pneumococcal conjugate vaccination coverage in children aged 2-59 months in Malawi. Hum Vaccin Immunother 2020 June 23 (Epub ahead of print).

DOI: $10.1056 /$ NEJMc2026544

DR. JEHAN AND COlleagues REPLY: Pittet et al. highlight the conundrum of pneumonia diagnosis in young children. There is no simple way to establish the cause and no reliable standard for imaging, ${ }^{1}$ even less so in low- and middle-income settings. Therefore, we took a treatment-centered approach in our trial (RETAPP), with the underlying assumption that most infections are viral and treated safely without antibiotics. ${ }^{2,3}$

Approximately $61 \%$ of the children in our trial had received age-appropriate doses of the pneumococcal conjugate vaccine, but the incidence of treatment failure was similar regardless of vaccination status $(5.4 \%$ among the unvaccinated children vs. $4.4 \%$ among the vaccinated children in the placebo group $[\mathrm{P}=0.34]$ and $2.1 \%$ vs. $2.8 \%$ in the antibiotic group $[\mathrm{P}=0.33])$. These results reaffirm the likelihood that mild pneumonia is most often viral and, thus, the likelihood that antibiotics constitute overtreatment.

In our trial, the presence of fever and a higher tertile of respiratory rate at presentation were predictors of treatment failure. The current collaboration between the WHO and the Bill and Melinda Gates Foundation to identify better predictors of nonsevere pneumonia may provide better case definitions to identify the patients most at risk for deterioration. 
Fyezah Jehan, M.B., B.S.

Imran Nisar, M.B., B.S.

Arjumand Rizvi, M.Sc.

Aga Khan University

Karachi, Pakistan

fyezah.jehan@aku.edu

Since publication of their article, the authors report no further potential conflict of interest.

1. Feikin DR, Hammitt LL, Murdoch DR, O'Brien KL, Scott JAG. The enduring challenge of determining pneumonia etiology in children: considerations for future research priorities. Clin Infect Dis 2017;64:Suppl 3:S188-S196.

2. Jehan F, Nisar I, Kerai S, Brown N, Ambler G, Zaidi AKM. Should fast breathing pneumonia cases be treated with antibiotics? The scientific rationale for revisiting management in low and middle income countries. Int J Infect Dis 2019;85:64-6.

3. Jehan F, Imran Nisar M, Kerai S, et al. A double blind community-based randomized trial of amoxicillin versus placebo for fast breathing pneumonia in children aged 2-59 months in Karachi, Pakistan (RETAPP). BMC Infect Dis 2016;16:13.

DOI: 10.1056/NEJMc2026544

\section{Triple Inhaled Therapy in COPD}

TO THE EDITOR: In reporting the results of the Efficacy and Safety of Triple Therapy in Obstructive Lung Disease (ETHOS) trial, Rabe et al. (July 2 issue) ${ }^{1}$ conclude that therapy with budesonide, glycopyrrolate, and formoterol was superior to combination therapy with glycopyrrolate-formoterol or budesonide-formoterol with regard to the annual rate of chronic obstructive pulmonary disease (COPD) exacerbations, symptoms, and health-related quality of life among patients with moderate-to-very-severe COPD. We note that approximately $75 \%$ of the participants in this trial received an inhaled glucocorticoid-containing regimen. In many of the patients assigned to the glycopyrrolate-formoterol group, inhaled glucocorticoid therapy was abruptly discontinued at the time of randomization (an action that might have led to COPD exacerbations). ${ }^{2,3}$

Thus, there are potential biases in the observed end points and clinically significant harmful effects of withdrawal of inhaled glucocorticoids in many patients. Can the authors comment on whether this aspect of trial design probably resulted in a false exaggeration of benefit with triple therapy as compared with glycopyrrolate-formoterol?

\section{Zhongbo Chen, M.D. \\ Zaichun Deng, M.D. Qunli Ding, M.D.}

Affiliated Hospital of Medical School of Ningbo University Ningbo, China

ccding2005@163.com

No potential conflict of interest relevant to this letter was reported.

1. Rabe KF, Martinez FJ, Ferguson GT, et al. Triple inhaled therapy at two glucocorticoid doses in moderate-to-very-severe COPD. N Engl J Med 2020;383:35-48.

2. Suissa S, Ernst P, Vandemheen KL, Aaron SD. Methodological issues in therapeutic trials of COPD. Eur Respir J 2008;31: 927-33.

3. Suissa S, Drazen JM. Making sense of triple inhaled therapy for COPD. N Engl J Med 2018;378:1723-4.

DOI: 10.1056/NEJMc2026881

TO THE EDITOR: Rabe et al. found that triple therapy with budesonide, glycopyrrolate, and formoterol was associated with a lower rate of moderate or severe COPD exacerbations than glycopyrrolate-formoterol or budesonide-formoterol. In addition, they found that high-dose budesonide-based triple therapy was associated with lower all-cause mortality than glycopyrrolateformoterol, which has been shown only in a single previous trial. ${ }^{1}$

A high percentage of patients in the ETHOS trial (29.8 to $31.6 \%$ ) had bronchodilator reversibility. The mean bronchodilator reversibility was not reported in the article about the ETHOS trial, but in the TRINITY and TRIBUTE trials,,$^{2,3}$ which did not show a survival benefit with triple therapy, the mean bronchodilator reversibility in the patients studied was 8 to $9 \%$. Is the survival benefit with high-dose budesonide-based triple therapy in this trial a result of the large percentage of enrolled patients with bronchodilator reversibility?

Chih-Cheng Lai, M.D.

Kaohsiung Veterans General Hospital

Tainan, Taiwan

dtmed141@gmail.com

No potential conflict of interest relevant to this letter was reported. 